- Empowerment meetings have resulted in patients and guardians feeling more informed about the care they receive and more in control.

- Focus group discussions with beneficiaries have resulted in changing practice at the services themselves.

- Empowerment meetings have been most successful when participants identify their own key concerns and have the space to explore solutions with others.

- Experience sharing and testimonies have played a great part in encouraging and learning from each other.

- The workshop with the project beneficiaries and Minister for Health allowed policy makers to hear first hand the experience of those living with life-limiting illnesses resulted in the minister pledging support to children's palliative care in Malawi.

The project is of interest to others who are working to support development of children's palliative care. We would like to share our approach and learning with others, and to encourage discussion about participation and empowerment in any palliative care setting.

\section{P107 WHO HAS DREAMS FULFILLED AT END OF LIFE? AN AUDIT OF A DREAM FULFILMENT ORGANISATION FOR CHILDREN AND YOUNG PEOPLE}

1,2 Jayne Galinsky, ${ }^{2}$ Liz Forbat, ${ }^{2}$ Emma France, ${ }^{1}$ Jane White. ${ }^{1}$ Cancer Care Research Centre, University of Stirling, Scotland, ${ }^{2}$ Dreams Come True - children and young people's charity

\subsection{6/bmjspcare-2013-000591.129}

Dream fulfilment provides a positive focus, hope and a sense of future for children and young people whose lives may be filled with hospital visits, pain or worry. This paper will present the results of an audit of a dream fulfilment charity's database. The audit systematically analyses user demographics to find out more about who uses charities such as these. This project fits with the strategic exercise conducted by the NHS in England to 'map' palliative care services across England so that both health professionals and the public can see what services are, and where they are available.

The data reported in this paper are drawn from Dreams Come True's records of children and young people with life limiting and long term conditions and their families, and the dreams that they have had.

Descriptive statistics are used to summarise the clinical and socio-demographic characteristics of the children/ families who have used the charity's services over the last five years. Results highlight the range of health conditions that children who use the charity have, as well as reporting on other factors such as the child's age, family size, gender and the type of dream requested. Analysis also suggests gaps in service reach which may reflect the unmet need in the sector as a whole.

Results will be of interest to health care professionals working in the fields of paediatric palliative care, and care of children with long term conditions, as well as to other dream fulfilment charities. The audit's findings have implications for referring organisations, such as hospices, and potential lessons for the adult sector.

\section{P108 THE TRANSITION TASKFORCE - MAKING THE DIFFERENCE FOR YOUNG PEOPLE WITH LIFE-LIMITING CONDITIONS}

\section{David Strudley, Lizzie Chambers. Together for Short Lives Transition Taskforce}

\subsection{6/bmjspcare-2013-000591.130}

Background Context Recent research from Leeds University shows there is a far greater number of young people who would benefit from palliative care, than previously believed. The study shows that the number of young people aged 16-19 with lifelimiting conditions is growing and has increased by $44.8 \%$ over the past 10 years. Services, including hospices, do not currently serve this growing population of young people and their families well.

Aim The project aims to make a real and tangible difference to the experience of young people with life-limiting conditions as they make the transition to adult services.

Approach Used Building on the growing evidence base, in particular findings from the STEPP Project, the project will adopt a two-pronged action-focussed approach to putting research and policy into practice:

- At national level, developing guidance, raising awareness, providing training and support for adult providers ensuring that the 'push' from children's services is balanced by a 'pull' from adult services.

- At local/regional level, supporting the development of services and partnerships between children's and adult services, so that the research and good practice that exists is implemented on the ground and disseminated nationally.

Outcomes The outcomes of the project will be that:

- young people and their families have more choice and control to make informed decisions about their care

- children's and adult services in the voluntary and statutory sector work together to support young people through transition

- wider services, such as housing, education, employment and independent living provide services that meet the needs of young people with life-limiting conditions

Application to Hospice Practice Adult hospice services can learn how to plan and deliver the care that young adults with life-limiting conditions need.

\section{P109 TRANSITION IN PALLIATIVE CARE STUDY DAY SERIES}

${ }^{1}$ Victoria Lidstone, ${ }^{2}$ Stephanie Sivell, ${ }^{3}$ Mark Taubert, ${ }^{4}$ Catherine Thompson, ${ }^{2}$ Annmarie Nelson. ${ }^{1}$ Cardiff and Vale University Health Board, ${ }^{2}$ Marie Curie Palliative Care Research Centre, Cardiff University School of Medicine, Cardiff, UK, ${ }^{3}$ Marie Curie Hospice, Cardiff and the Vale, ${ }^{4} T$ Hafan

\subsection{6/bmjspcare-2013-000591.13}

Background / Context Medical advances have led to more children with life-limiting conditions surviving into adulthood with an increasing number accessing adult Specialist Palliative Care (SPC) services. These young adults often have conditions unfamiliar to adult SPC professionals, who may be inexperienced in caring for their complex needs. Results from previous research carried out by the same team, identified a need for a training/ education programme for adult SPC professionals and provided recommendations regarding content and delivery.

Aim To develop a training programme to help up skill and build confidence in adult SPC professionals regarding the care of young adults with life-limiting conditions. 
Approach Used A project team, consisting of children's and adult palliative care providers, researchers and the All Wales Palliative Care Transition Lead, was convened to design a purposeful and effective training. The training was specifically created such that it would be suitable for a multi-disciplinary audience which would provide a comprehensive grounding if completed, but could also be delivered piecemeal so that participants had as much flexibility as possible regarding attendance. The content was informed by previous research.

Outcomes A series of six linked study days was designed such that each day could be attended as standalone, but those attending all of the days would have a comprehensive grounding in young adult care. Each study day includes a balance of clinical, practical and psychosocial topics suitable for a multi-disciplinary audience. Training will be delivered between June 2013 and June 2014.

Application to Hospice Practice The study day series is open to all professionals working in adult hospices who may increasingly be called upon to care for young people with life-limiting conditions. By improving the knowledge and skill base of these professionals, professional confidence will increase and lead to an improvement of care for these young people.

\section{P110 EVALUATION OF MEDICINES ADHERENCE IN DAY HOSPICE PATIENTS}

Julia Greenwood. St Gemma's Hospice, Leeds, England

10.1136/bmjspcare-2013-000591.132

Background Central to the care of patients in Day Hospice is an understanding of their compliance with medication. The Hospice has a Medicines Management Policy in place, but had no structured approach to measuring patients' levels of concordance, from their perspective.

Aim of the Evaluation The aim of the evaluation was to develop a method by which patient compliance could be assessed, explored and improved

Method The National Institute for Clinical Excellence (NICE) produced guidance and a patient questionnaire on Medicines Adherence in 2009. The questions explore the role of the healthcare professional in supporting patient's decision making, and understanding of their medicines. An amended version of the NICE questionnaire was developed and used, for which sixteen patients were randomly selected. Verbal consent was obtained and anonymity assured.

Results It provided useful information about our effectiveness in supporting patients with their medicines adherence. We scored well in engaging patients in joint decision-making, however some other aspects such as exploration of the burdens and benefits of medication, and common side effects needed improvement.

Limitations

Some questions could be misinterpreted, and there was no facility to expand on answers given. The length and style of the questionnaire was quite difficult to implement with people who were fatigued and unwell.

Conclusions This evaluation has been important to our understanding of our effectiveness in supporting medicines adherence. We intend to inform NICE of the amendments made prior to using the questionnaire within specialist palliative day services. We aim to further develop the questionnaire to evaluate patients' experiences and concerns regarding their medication regimes, and to address the need for carer involvement (End of Life Care Strategy 2008), as they underpin concordance for many of our patients.

\section{P111 TERMINAL OPIOID AND SEDATIVE TITRATION IN TWO HOSPICES}

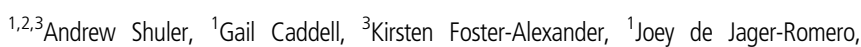
${ }^{2,3,4}$ Marlise Poolman. ${ }^{1}$ Nightingale House Hospice, Wrexham, Wales, ${ }^{2}$ Betsi Cadwaladr University Health Board, ${ }^{3}$ St Davids Hospice, LLandudno, WalesBangor University, Wales, ${ }^{4}$ Bangor University, Wales

\subsection{6/bmjspcare-2013-000591.133}

Introduction There was anecdotal observation by professionals working across both sites that there was a difference to practice with opioid and sedative titration at the end of life. As an extension to early work at one of the hospices, it was decided to consider practice across the region, so a second hospice was invited to participate.

Aims To quantitively assess practice of sedative use in relation to the EAPC recommended framework for the use of sedation in palliative care.

To quantitively assess practice of opioid use in the terminal phase.

To assess whether practice differs between hospices in North Wales, and to consider any potential reasons for the difference and implications for practice.

Methods A retrospective case-note review of patients who died in the two units. Data collected included drugs, doses and increments, and proxy assessment of symptoms in the last week of life (MSAS-GDI).

Results Groups were comparable in terms of background and demographics.

Practice differed with one unit using Midazolam and Levomepromazine more frequently and at higher starting doses.

There was no difference to symptom burden between sites.

Discussion All doses used were within the limits described in the EAPC framework.

At subsequent focus group discussion potential reasons were discussed including differences in anti-secretory medication use and its impact on sedative use.

These findings have precipitated further work on both sites.

\section{P112 MORPHINE AND OTHER OPIOID PAINKILLERS FOR MODERATE TO SEVERE PAIN: A NICE GUIDANCE COMPLIANT PATIENT INFORMATION LEAFLET}

${ }^{1,2}$ Ray Bunn, ${ }^{2}$ Amanda Gregory. ${ }^{1}$ Kamson's Pharmacy, Crawley, UK, ${ }^{2}$ St. Catherine's Hospice, Crawley, UK

\subsection{6/bmjspcare-2013-000591.134}

The Medicines Management Group (a multidisciplinary team comprising of medical, nursing and pharmacy representation) at an independent hospice has developed a patient information leaflet (PIL) on strong opioids. This decision was made in response to the recent National Institute for Health and Clinical Excellence (NICE) guidance on 'Opioids in palliative care: safe and effective prescribing of strong opioids for pain in palliative care of adults' ${ }^{(1)}$ which recommends that verbal communication between healthcare professionals and patients about their medicines should be supported by evidence based, written information. The aim was to produce a PIL which was NICE guidance 